\title{
Sensitivity, Optimal Control, and Cost-Effectiveness Analysis of Intervention Strategies of Filariasis
}

\author{
Darmawati $^{*}$, Musafira $^{2}$, Darma Ekawati ${ }^{3}$, Wahyudin Nur $^{4}$, Muhlis $^{5}$, Siti \\ Fatima Azzahra ${ }^{6}$ \\ 1,2,3,4,5 Department of Mathematics, Universitas Sulawesi Barat, Majene, Indonesia \\ ${ }^{6}$ Department of Nursing, Universitas Sulawesi Barat, Majene, Indonesia \\ *Corresponding author. Email: darmath@unsulbar.ac.id
}

\begin{abstract}
In this work, sensitivity, optimal control, and cost-effectiveness of several intervention strategies of filariasis are discussed. We study the intervention strategies that are related to bednet use, insecticide, and the combination of bed-net use and insecticide. We use Pontryagin's maximum principle to characterize the optimal controls. The Average Cost-Effectiveness Ratio (ACER) and Infection Averted Ratio (IAR) are used to identify the most cost-effective strategy. We also determine the basic reproduction number and investigate the sensitivity of the basic reproduction number on the parameters that are related to bed-net use and insecticide. Based on the ACER values, the most cost-effective strategy to control filariasis is insecticide intervention. On the other hand, the IAR values indicates that bed-net use intervention is the most cost-effective strategy. Furthermore, it is also the most effective strategy to eliminate filariasis. The sensitivity analysis results show that the control parameter related to bed net use and treatment have a central role in reducing the basic reproduction number and filariasis spread.
\end{abstract}

Keywords:

Optimal Control; Sensitivity Analysis; Cost-Effectiveness Analysis; Filariasis

How to Cite:

D. Darmawati, M. Musafira, D. Ekawati, W. Nur, M. Muhlis, and S. F. Azzahra, "Sensitivity, Optimal Control, and Cost-Effectiveness Analysis of Intervention Strategies of Filariasis", Jambura J. Math., vol. 4, No. 1, pp. 64-76, 2022, doi: https://doi.org/10.34312/jjom.v4i1.11766

\section{Introduction}

Mosquito-borne diseases such as malaria, dengue fever, chikungunya, and zika cause more than one million deaths every year. Unlike some of the mosquito -borne diseases previously mentioned, filariasis is a mosquito-borne disease which can cause permanent disability [1]. Filariasis is a chronic infectious disease caused by filarial worms and transmitted to humans by infected mosquitoes [2]. More than 120 million people are affected by filarial worm infection in 83 tropical and subtropical countries in several regions, i.e., Southeast Asia, Africa, the Mediterranean, the Western Pacific, and the Americas are endemic areas [3]. In Indonesia, filariasis is an endemic disease in several provinces, e.g., West Sulawesi [4].

e-ISSN: 2656-1344 (C) 2022 D. Darmawati, M. Musafira, D. Ekawati, W. Nur, M. Muhlis, S. F. Azzahra| Under the license CC BY-NC 4.0

Received: 15 October 2021 | Accepted: 21 November 2021 | Online: 1 January 2022 
The implementation of the filariasis elimination program is carried out by several methods, namely providing mass drug therapy, preventing and limiting disability, controlling vectors, and developing research related to filariasis. Another intervention usually applied in managing the spread of filariasis is to reduce contact between humans and mosquitoes [3].

In recent years, the spread of filariasis has been studied using mathematical models [5-9]. Supriatna et al. [5] developed a mathematical model related to the spread of filariasis to evaluate the long-term impact of treatment of patients with filariasis in West Java, Indonesia. The outcomes of this research are the provision of additional care, controlling the number of contact between humans and mosquitoes, and controlling the mosquito population can reduce the cases of filariasis. Bhunu et al. [6] developed a mathematical model of filariasis that considers humans that are still in the incubation period. They found that treatment for elephantiasis patients can reduce the number of filariasis cases. However, filariasis cannot be eliminated if the treatment is only given to humans who have shown clinical symptoms, such as leg swelling (elephantiasis). They suggest that humans who are still in the incubation period should be treated so that the disease can be eradicated. The filariasis model developed to assess the impact of quarantine is discussed in [7]. The result of this study is that treatment for humans who are suffering from filariasis is more effective in reducing filariasis cases when compared to quarantine interventions.

To study the impact of reducing the possibility of the contact between human and mosquito which can disturb transmission process from mosquitoes to humans and from humans to mosquitoes, we considered bed-net use intervention. The treatment that can reduce the filarial parasite in the blood of treated humans was also considered in our model because we wanted to study the impact of reducing the rate of infection from humans to mosquitoes. Furthermore, to investigate the impact of controlling mosquito populations, we also pay attention to the use of insecticides. Hence, in contrast to the previously mentioned studies, we develop a filariasis model with the use of bed-net, treatment, and the use of insecticides. We perform sensitivity analysis to investigate normalized forward sensitivity index [10] of several parameters. Pontryagin's maximum principle is used to solve the optimal control problem. The cost-effectiveness of several intervention strategies of filariasis are also studied by using similar methods used in [11-14].

\section{Methods}

The study is conducted by doing the following steps:

1. Formulating a mathematical model of filariasis that considers the use of bed-nets and insecticides.

2. Identifying the equilibrium points of the model and the conditions for its existence.

3. Determining the basic reproduction number using the next-generation matrix.

4. Performing sensitivity analysis.

5. Determining the solution of the optimal control problem using Pontryagin's maximum principle.

6. Conducting cost-effectiveness analysis by calculating and comparing average costeffectiveness ratio (ACER) and infection averted ratio (IAR) for each intervention scheme. According to [15], we can calculate ACER and IAR by using the following formulas, respectively. 


$$
\begin{aligned}
\text { ACER } & =\frac{\text { Cost produced by the intervention }}{\text { Number of Infection Averted }} \\
\text { IAR } & =\frac{\text { Number of infection averted }}{\text { Number of recovered }}
\end{aligned}
$$

\section{Results and Discussion}

\subsection{Model Formulation}

In this section, we describe the process of model formulation. Figure 1 shows the flow diagram of compartmens. Human population is divided into four compartments, i.e., susceptible human $\left(S_{h}\right)$, latent human $\left(E_{h}\right)$, infectious human $\left(I_{h}\right)$, and treated human $\left(T_{h}\right)$. On the other hand, we divide the mosquito population into two disjoint compartments, i.e., susceptible mosquito $\left(S_{m}\right)$ and infectious mosquito $\left(I_{m}\right)$.

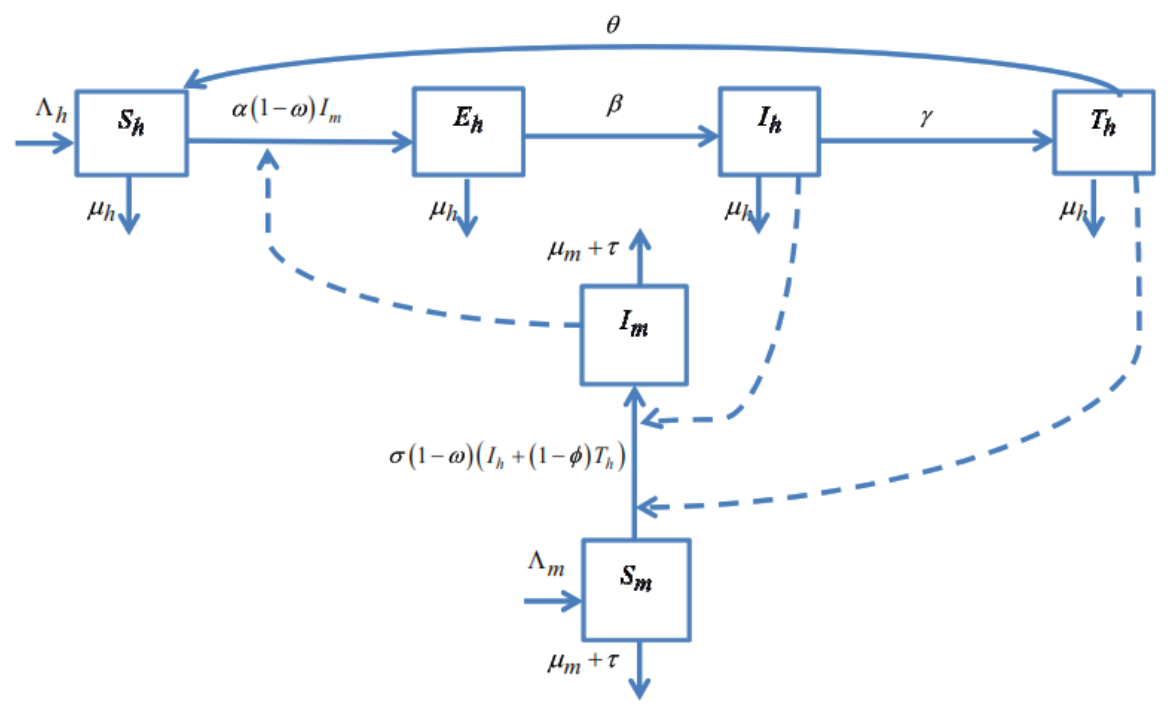

Figure 1. Flow Diagram of Compartments

The total number of susceptible humans increases with constant recruitment which is denoted by $\Lambda_{h}$. Here, we assume that treated humans who recover from filariasis do not get immunity. Hence, as a result of treated humans who recover from filariasis at rate $\theta$, the total number of susceptible humans increases by $\theta T_{h}$. The total number of susceptible humans decreases by $\alpha(1-\omega) S_{h} I_{m}$ as a consequence of new infection that occurs after adequate contact between susceptible humans and infectious mosquitoes. Here, $\alpha$ is the transmission rate from infectious mosquitoes to susceptible humans and $\omega$ is the proportion of humans who use bed-net at night. Because of the natural death of susceptible humans, the total number of susceptible humans decreases by $\mu_{h} S_{h}$. Here, $\mu_{h}$ is the natural death rate of human. Therefore, we get the following ordinary differential equation that describe the dynamics of susceptible humans.

$$
\frac{d S_{h}}{d t}=\Lambda_{h}-\alpha(1-\omega) S_{h} I_{m}+\theta T_{h}-\mu_{h} S_{h} .
$$

Latent humans are humans who are infected but have not been able to transmit the disease to mosquitoes. The total number of latent humans increases by new infection, 
that is $\alpha(1-\omega) S_{h} I_{m}$. After the latent period $\beta^{-1}$ ends, the latent human becomes an infectious human. Hence, the total number of latent humans decreases by $\beta E_{h}$. Moreover, due to the natural death of latent humans, the total number of latent humans also decreases by $\mu_{h} E_{h}$. Thus, we have

$$
\frac{d E_{h}}{d t}=\alpha(1-\omega) S_{h} I_{m}-\beta E_{h}-\mu_{h} E_{h}
$$

The total number of infectious humans increases by new infectious humans, that is $\beta E_{h}$. Because of the natural death of humans, the total number of infectious humans decreases by $\mu_{h} I_{h}$. Here, we assume that the average waiting time of infectious humans to get treatment is $\gamma^{-1}$. Hence, the total number of infectious humans decreases by $\gamma I_{h}$. Consequently, we get

$$
\frac{d I_{h}}{d t}=\beta E_{h}-\gamma I_{h}-\mu_{h} I_{h} .
$$

The total number of treated humans increases by $\gamma I_{h}$ and decreases by the natural death of treated human $\mu_{h} T_{h}$. The following ordinary differential equation represents the dynamics of treated humans.

$$
\frac{d T_{h}}{d t}=\gamma I_{h}-\theta T_{h}-\mu_{h} T_{h}
$$

The total number of susceptible mosquitoes increases by constant recruitment, that is $\Lambda_{m}$. After sufficient contact with infectious humans and treated humans, susceptible mosquitoes get infected. Hence, the total number of susceptible mosquitoes decreases by $\sigma(1-\omega)\left(I_{h}+(1-\phi) T_{h}\right) S_{m}$. $\phi$ is the parameter that measures the effectiveness of treatment in reducing the transmission from treated humans to mosquitoes. If $\phi=1$, then treated humans can not transmit the disease. If $\phi=0$, then the treated humans can transmit the disease similar to infectious humans who do not get treatment. If $\phi \in(0,1)$, then the treatment successfully reduces the transmission from treated humans to susceptible mosquitoes. Here, we assume that the natural death rate of mosquitoes is $\mu_{m}$ and the insecticide-related death rate of mosquitoes is $\tau$. So, the total number of susceptible mosquitoes decreases by $\mu_{m} S_{m}+\tau S_{m}$. As a result, we have

$$
\frac{d S_{m}}{d t}=\Lambda_{m}-\sigma(1-\omega)\left(I_{h}+(1-\phi) T_{h}\right) S_{m}-\mu_{m} S_{m}-\tau S_{m}
$$

The total number of infectious mosquitoes increases by new infection, that is $\sigma(1-\omega)\left(I_{h}+(1-\phi) T_{h}\right) S_{m}$. Similar to susceptible mosquitoes, the total number of infectious mosquitoes decreases by $\mu_{m} I_{m}+\tau I_{m}$. Accordingly, we get

$$
\frac{d I_{m}}{d t}=\sigma(1-\omega)\left(I_{h}+(1-\phi) T_{h}\right) S_{m}-\mu_{m} I_{m}-\tau I_{m}
$$

Based on the model formulation process above, we have the following system.

$$
\begin{aligned}
\frac{d S_{h}}{d t} & =\Lambda_{h}-\alpha(1-\omega) S_{h} I_{m}+\theta T_{h}-\mu_{h} S_{h}, \\
\frac{d E_{h}}{d t} & =\alpha(1-\omega) S_{h} I_{m}-\beta E_{h}-\mu_{h} E_{h}, \\
\frac{d I_{h}}{d t} & =\beta E_{h}-\gamma I_{h}-\mu_{h} I_{h},
\end{aligned}
$$




$$
\begin{aligned}
\frac{d T_{h}}{d t} & =\gamma I_{h}-\theta T_{h}-\mu_{h} T_{h}, \\
\frac{d S_{m}}{d t} & =\Lambda_{m}-\sigma(1-\omega)\left(I_{h}+(1-\phi) T_{h}\right) S_{m}-\mu_{m} S_{m}-\tau S_{m}, \\
\frac{d I_{m}}{d t} & =\sigma(1-\omega)\left(I_{h}+(1-\phi) T_{h}\right) S_{m}-\mu_{m} I_{m}-\tau I_{m} .
\end{aligned}
$$

\subsection{Reproduction Number}

The reproduction number is determined by using NGM (Next Generation Matrix) method that is presented in [16]. Firstly, we determine the disease free equilibrium point. After simple calculation process, we obtain the disease-free equilibrium point $P_{0}=\left(S_{h}^{0}, E_{h}^{0}, I_{h}^{0}, T_{h}^{0}, S_{m}^{0}, T_{m}^{0}\right)=\left(\frac{\Lambda_{h}}{\mu_{h}}, 0,0,0, \frac{\Lambda_{m}}{\mu_{m}+\tau}\right)$. Following the method given in [16], we determine $F$ and $V$. Here, $E_{h}, I_{h}, T_{h}, I_{m}$ are considered to be infected compartments. Therefore, we get

$$
\begin{aligned}
F \quad & =\left(\begin{array}{cccc}
0 & 0 & 0 & \alpha(1-\omega) \frac{\Lambda_{h}}{\mu_{h}} \\
0 & 0 & 0 & 0 \\
0 & 0 & 0 & 0 \\
0 & \sigma(1-\omega) \frac{\Lambda_{m}}{\mu_{m}+\tau} & \sigma(1-\omega)(1-\phi) \frac{\Lambda_{m}}{\mu_{m}+\tau} & 0
\end{array}\right), \\
V & =\left(\begin{array}{cccc}
\beta+\mu_{h} & 0 & 0 & 0 \\
-\beta & \gamma+\mu_{h} & 0 & 0 \\
0 & -\gamma & \theta+\mu_{h} & 0 \\
0 & 0 & 0 & \mu_{m}+\tau
\end{array}\right) .
\end{aligned}
$$

It is straightforward to prove that the spectral radius of $F V^{-1}$ is

$$
\rho\left(F V^{-1}\right)=\sqrt{\frac{\alpha(1-\omega) \Lambda_{h}}{\left(\mu_{m}+\tau\right) \mu_{h}} v_{1}}
$$

where

$$
v_{1}=\frac{\sigma(1-\omega) \beta \Lambda_{m}}{\left(\beta+\mu_{h}\right)\left(\gamma+\mu_{h}\right)\left(\mu_{m}+\tau\right)}+\frac{\sigma(1-\omega)(1-\phi) \beta \gamma \Lambda_{m}}{\left(\beta+\mu_{h}\right)\left(\gamma+\mu_{h}\right)\left(\theta+\mu_{h}\right)\left(\mu_{m}+\tau\right)} .
$$

Therefore, the reproduction number is given as follows

$$
R_{0}=\sqrt{\frac{\alpha(1-\omega)^{2} \Lambda_{h} \sigma \beta \Lambda_{m}\left(\theta+\mu_{h}+(1-\phi) \gamma\right)}{\left(\mu_{m}+\tau\right)^{2}\left(\beta+\mu_{h}\right)\left(\gamma+\mu_{h}\right)\left(\theta+\mu_{h}\right) \mu_{h}}} .
$$

Based on Theorem 2 in [16], we obtain the following Theorem.

Theorem 1. The disease free equilibrium point $P_{0}$ is stable if $R_{0}<1$. On the other hand, $P_{0}$ is unstable if $R_{0}>1$.

\subsection{Sensitivity Analysis}

In this section, we perform sensitivity analysis to identify the effect of several parameters, e.g., $\Lambda_{m}, \tau, \omega, \phi$, on the basic reproduction number. We determine the normalized forward sensitivity index that is described in [10]. The sensitivity index of 
each parameter is given as

$$
\begin{aligned}
Y_{\Lambda_{m}}^{R_{0}} & =\frac{\partial R_{0}}{\partial \Lambda_{m}} \times \frac{\Lambda_{m}}{R_{0}}=\frac{1}{2}>0, \\
Y_{\tau}^{R_{0}} & =\frac{\partial R_{0}}{\partial \tau} \times \frac{\tau}{R_{0}}=\frac{-\tau}{\tau+\mu_{v}}<0, \\
Y_{\phi}^{R_{0}} & =\frac{\partial R_{0}}{\partial \phi} \times \frac{\phi}{R_{0}}=\frac{-\phi \gamma}{\theta+\mu_{h}+\gamma(1-\phi)}<0, \\
Y_{\omega}^{R_{0}} & =\frac{\partial R_{0}}{\partial \omega} \times \frac{\omega}{R_{0}}=\frac{-\omega}{1-\omega}<0 .
\end{aligned}
$$

$\mathrm{Y}_{\Lambda_{m}}^{R_{0}}=0.5$ shows that $1 \%$ increase in $\Lambda_{m}$ causes $0.5 \%$ increase in $R_{0}$. Hence, we can reduce the basic reproduction number by reducing the recruitment rate of mosquitoes. $\mathrm{Y}_{\tau}^{R_{0}}=\frac{-\tau}{\tau+\mu_{v}}$ means that $1 \%$ increase in $\tau$ will produce $\frac{\tau}{\tau+\mu_{v}}$ decrease in $R_{0}$. Therefore, the basic reproduction number decreases as $\tau$ increases. However, the magnitude of the decrease in $R_{0}$ depends on the baseline value of $\tau$ and $\mu_{v}$. To determine the sensitivity index of the parameters, we use the parameter values presented in Table 1 as the baseline of parameter values.

Table 1. Parameter Values

\begin{tabular}{cccc}
\hline Parameter & Values & Unit & Source \\
\hline$\Lambda_{h}$ & $\frac{500 \times 1.98}{365 \times 100}$ & human $\times$ day $^{-1}$ & Estimated \\
$\Lambda_{m}$ & 6 & mosquitoes $\times$ day $^{-1}$ & {$[7]$} \\
$\mu_{h}$ & $\frac{1}{65 \times 365}$ & day $^{-1}$ & {$[17]$} \\
$\mu_{m}$ & $\frac{1}{13}$ & day $^{-1}$ & {$[18],[19]$} \\
$\alpha$ & $0.01 \times 0.01$ & mosquito $^{-1} \times$ day $^{-1}$ & {$[7]$} \\
$\sigma$ & $1.52 \times 0.01$ & human $^{-1} \times$ day $^{-1}$ & {$[8]$} \\
$\beta$ & $\frac{1}{6 \times 4 \times 7}$ & day $^{-1}$ & {$[20]$} \\
$\gamma$ & $\frac{1}{7}$ & day $^{-1}$ & {$[4]$} \\
$\theta$ & $\frac{1}{6 \times 7}$ & day $^{-1}$ & {$[3]$} \\
$\tau$ & 0.001 & day $^{-1}$ & Assumed \\
$\omega$ & $20 \%$ & & Assumed \\
$\phi$ & $20 \%$ & & {$[21],[22]$} \\
\hline
\end{tabular}
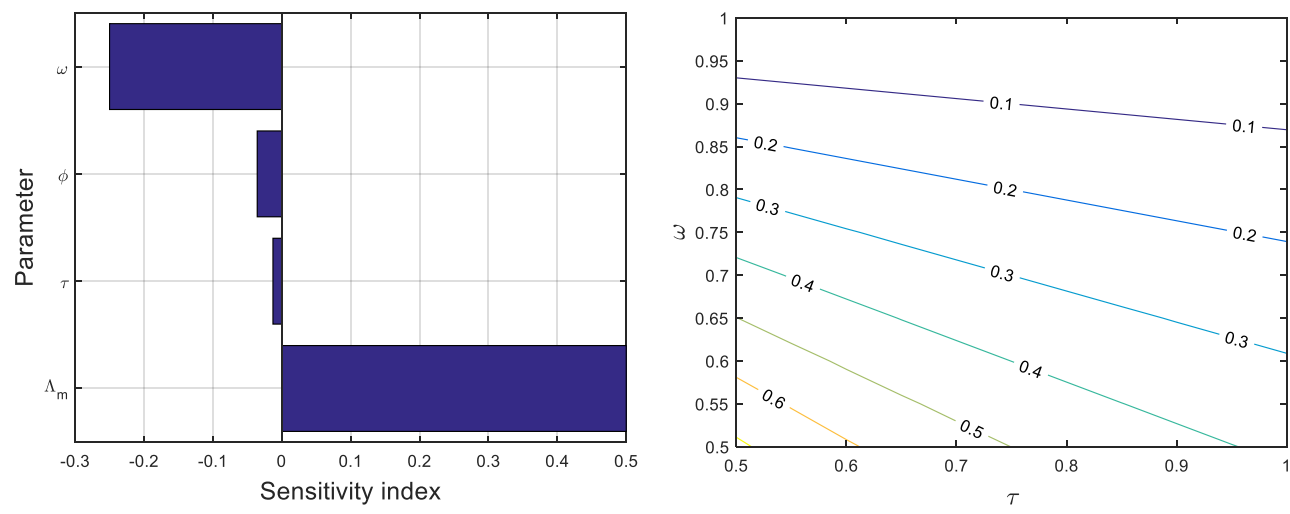

Figure 2. Sensitivity of $R_{0}(\mathrm{a})$ and relationship between $\tau$, $\omega$, and $R_{0}(\mathrm{~b})$

After substituting the parameter values, we obtain $\mathrm{Y}_{\Lambda_{m}}^{R_{0}}=0.500, \mathrm{Y}_{\tau}^{R_{0}}=0.013$, $Y_{\phi}^{R_{0}}=-0.036$, and $Y_{\omega}^{R_{0}}=0.250$. These results show that we must control the growth of 
mosquito population. Moreover, the use of bed-net and treatment which can reduce the filarial worm in the blood of treated humans have a central role in filariasis control. relationship between $\tau, \omega$, and $R_{0}$ can be seen in Figure 2 .

\subsection{Optimal Control Problem}

For the optimal control model, we set $\omega=u_{1}$ and $\tau=u_{2}$. Therefore, the optimal control model is given as follows

$$
\begin{aligned}
\frac{d S_{h}}{d t} & =\Lambda_{h}-\alpha\left(1-u_{1}\right) S_{h} I_{m}+\theta T_{h}-\mu_{h} S_{h} \\
\frac{d E_{h}}{d t} & =\alpha\left(1-u_{1}\right) S_{h} I_{m}-\beta E_{h}-\mu_{h} E_{h}, \\
\frac{d I_{h}}{d t} & =\beta E_{h}-\gamma I_{h}-\mu_{h} I_{h}, \\
\frac{d T_{h}}{d t} & =\gamma I_{h}-\theta T_{h}-\mu_{h} T_{h} \\
\frac{d S_{m}}{d t} & =\Lambda_{m}-\sigma\left(1-u_{1}\right)\left(I_{h}+(1-\phi) T_{h}\right) S_{m}-\mu_{m} S_{m}-u_{2} S_{m}, \\
\frac{d I_{m}}{d t} & =\sigma\left(1-u_{1}\right)\left(I_{h}+(1-\phi) T_{h}\right) S_{m}-\mu_{m} I_{m}-u_{2} I_{m} .
\end{aligned}
$$

Our purposes are to determine the control strategy that minimizes the number of infectious humans and latent humans as well as the costs of interventions. Hence, we intend to examine the control strategy that minimizes the following objective function

$$
\mathrm{O}\left(u_{1}, u_{2}\right)=\int_{0}^{T}\left(D_{1} E_{h}+D_{2} I_{h}+D_{3} u_{1}^{2}+D_{4} u_{2}^{2}\right) d t
$$

subject to system (4). $D_{1}$ and $D_{2}$ are the relative cost weight for $E_{h}$ and $I_{h}$, respectively. $D_{3}$ and $D_{4}$ are the relative cost weight associated with bed-net use and insecticide, respectively. The final time is $T$. We will determine $u_{1}^{+}, u_{2}^{+}$, such that

$$
\mathrm{O}\left(u_{1}^{+}, u_{2}^{+}\right)=\min _{U}\left(u_{1}, u_{2}\right),
$$

where

$$
U=\left\{u_{1}:[0, T] \rightarrow\left[0, u_{1}^{\max }\right], u_{2}:[0, T] \rightarrow\left[0, u_{2}^{\max }\right] \text {, are Lebesgue measurable }\right\} .
$$

We solve the optimal control problem by applying Pontryagin's maximum principle. Based on the objective function (5) and the optimal control model (4), we establish the Hamiltonian function $\mathrm{H}$ as follows

$$
\begin{aligned}
\mathrm{H}= & D_{1} E_{h}+D_{2} I_{h}+D_{3} u_{1}^{2}+D_{4} u_{2}^{2} \\
& +w_{1}\left[\Lambda_{h}-\alpha\left(1-u_{1}\right) S_{h} I_{m}+\theta T_{h}-\mu_{h} S_{h}\right] \\
& +w_{2}\left[\alpha\left(1-u_{1}\right) S_{h} I_{m}-\beta E_{h}-\mu_{h} E_{h}\right] \\
& +w_{3}\left[\beta E_{h}-\gamma I_{h}-\mu_{h} I_{h}\right] \\
& +w_{4}\left[\gamma I_{h}-\theta T_{h}-\mu_{h} T_{h}\right] \\
& +w_{5}\left[\Lambda_{m}-\sigma\left(1-u_{1}\right)\left(I_{h}+(1-\phi) T_{h}\right) S_{m}-\mu_{m} S_{m}-u_{2} S_{m}\right] \\
& +w_{6}\left[\sigma\left(1-u_{1}\right)\left(I_{h}+(1-\phi) T_{h}\right) S_{m}-\mu_{m} I_{m}-u_{2} I_{m}\right] .
\end{aligned}
$$


where $w_{1}, w_{2}, \ldots, w_{6}$ are adjoint variables. Next, we determine the adjoint system. The adjoint system for the optimal control problem is given as follows

$$
\begin{aligned}
& \frac{d w_{1}}{d t}=-\frac{\partial \mathrm{H}}{\partial S_{h}}=\left(w_{1}-w_{2}\right) \alpha I_{m}\left(1-u_{1}\right)+w_{1} \mu_{h}, \\
& \frac{d w_{2}}{d t}=-\frac{\partial \mathrm{H}}{\partial E_{h}}=\left(w_{2}-w_{3}\right) \beta+w_{2} \mu_{h}-D_{1}, \\
& \frac{d w_{3}}{d t}=-\frac{\partial \mathrm{H}}{\partial I_{h}}=\left(w_{3}-w_{4}\right) \gamma+w_{3} \mu_{h}+\left(w_{5}-{ }_{6}\right) \sigma\left(1-u_{1}\right) S_{m}-D_{2}, \\
& \frac{d w^{4}}{d t}=-\frac{\partial \mathrm{H}}{\partial T_{h}}=\left(w_{4}-w_{1}\right) \theta+w_{4} \mu_{h}, \\
& \frac{d w_{5}}{d t}=-\frac{\partial \mathrm{H}}{\partial S_{m}}=w_{5}\left(u_{2}+\mu_{m}\right)+\left(w_{5}-w_{6}\right) \sigma\left(1-u_{1}\right)\left(I_{h}+(1-\phi) T_{h}\right), \\
& \frac{d w_{6}}{d t}=-\frac{\partial \mathrm{H}}{\partial I_{m}}=\left(w_{1}-w_{2}\right) \alpha\left(1-u_{1}\right) S_{h}+w_{6}\left(u_{2}+\mu_{m}\right),
\end{aligned}
$$

with boundary conditions $w_{i}(T)=0$ for $i=1, \ldots 6$. The derivative of Hwith respect to control parameters $u_{1}$ and $u_{2}$ are, respectively, given as follows

$$
\begin{aligned}
& \frac{\partial \mathrm{H}}{u_{1}}=2 D_{3} u_{1}+\left(w_{1}-w_{2}\right) \alpha I_{m} S_{h}+\left(w_{5}-w_{6}\right) \sigma S_{m}\left(I_{h}+(1-\phi) T_{h}\right), \\
& \frac{\partial \mathrm{H}}{u_{2}}=2 D_{4} u_{2}-w_{5} S_{m}-w_{6} I_{m} .
\end{aligned}
$$

After solving $\frac{\partial \mathrm{H}}{\partial u_{1}}=\frac{\partial \mathrm{H}}{\partial u_{2}}=0$, we get

$$
\begin{aligned}
& u_{1}^{*}=\frac{\left(w_{2}-w_{1}\right) \alpha I_{m} S_{h}+\left(w_{6}-w_{5}\right) \sigma S_{m}\left(I_{h}+(1-\phi) T_{h}\right)}{2 D_{3}}, \\
& u_{2}^{*}=\frac{w_{5} S_{m}+w_{6} I_{m}}{2 D_{4}} .
\end{aligned}
$$

Based on the upper and lower bound of $u_{1}$ and $u_{2}$, the optimal value $u_{1}^{+}$and $u_{2}^{+}$are, respectively, given by

$$
\begin{aligned}
& u_{1}^{+}=\min \left\{\max \left\{0, u_{1}^{*}\right\}, 1\right\} \\
& u_{2}^{+}=\min \left\{\max \left\{0, u_{2}^{*}\right\}, 1\right\} .
\end{aligned}
$$

\subsection{Effectiveness and Cost Effectiveness}

For the effectiveness and cost-effectiveness analysis, we adopt the following measures.

1. We regard infectious humans and latent humans as infected humans. Consequently, the total number of infected humans in the course of Tperiod with and without control are, respectively, given by

$$
T_{w c}=\int_{0}^{T}\left(E_{h}^{*}(t)+I_{h}^{*}(t)\right) d t, T_{w o c}=\int_{0}^{T}\left(E_{h}(t)+I_{h}(t)\right) d t .
$$

where $E_{h}^{*}$ and $I_{h}^{*}$ represent the number of latent humans and infectious humans when the control strategy is applied. Consequently, $T_{a v}=T_{w o c}-T_{w c}$ is the total number of infected humans avoided because of the implementation of control strategy. 
2. Following [13], we use $\bar{E}$ to measure the effectiveness of intervention.

$$
\bar{E}=\frac{T_{a v}}{T_{w o c}}
$$

3. Following [11], the Average Cost-Effectiveness Ratio (ACER) and the Infected Averted Ratio (IAR) are given by

$$
\begin{array}{r}
A C E R=\frac{T C}{T_{a v}}, \\
\text { IAR }=\frac{T_{a v}}{T_{r}},
\end{array}
$$

where TC $=\int_{0}^{T}\left(D_{3} u_{1}^{2}+D_{4} u_{2}^{2}\right) d t$ and $T_{r}=\int_{0}^{T} \theta T_{h} d t$ tare total cost of intervention and total number of recovered humans during $T$ period, respectively.

\subsection{Numerical Simulations}

The parameter values used are presented in Table 1 which gives $R_{0}=7.5196$. The initial condition used is $S_{h}(0)=100, E_{h}(0)=10, I_{h}(0)=5, S_{m}(0)=200, T_{h}(0)=30$, and $I_{m}(0)=30$. For the numerical study, we set $D_{1}=1, D_{2}=4, D_{3}=1$, and $D_{4}=3$. Here we assume that $u_{1}^{\max }=0.8$ and $u_{2}^{\max }=0.1$. We investigate the following strategy.

1. Strategy 1: The Effect of Bed-Net Use Intervention only.

In this part, we investigate the effects of the bed-net use intervention only. For the numerical simulations, we set $u_{1} \geq 0$ and $u_{2}=0$. Figure 3 shows that strategy 1 can reduce filariasis cases. Without a control strategy, the peak of the number of infected humans almost reaches 25 . Without control, the number of infected humans at the end of the period is 6 . When strategy 1 is implemented, the number of infected humans at the end of the period decreases to near 0. Moreover, when strategy 1 is applied, the peak of the number of infected humans is about 15 only. Figure 3 shows that the optimal value of $u_{1}$ should be always at its upper bound.
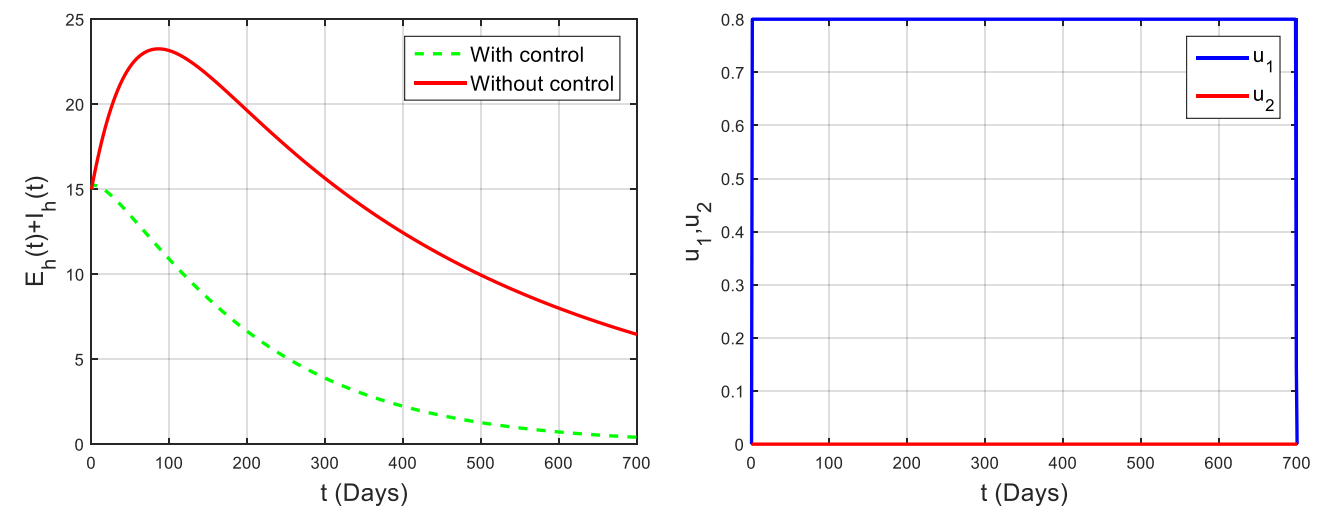

Figure 3. Dynamics of infected human and control profile when strategy 1 is applied

2. Strategy 2: The Effect of Insecticide Intervention only.

Now, we explore the effect of insecticide intervention only. We conduct the numerical simulations with $u_{1}=0$ and $u_{2} \geq 0$. Figure 4 shows that filariasis cases can be controlled when strategy 2 is implemented. If strategy 2 is implemented, the number 
of infected humans at the end of the period is 2 . Figure 4 shows that control $u_{2}$ should be always at its upper bound.
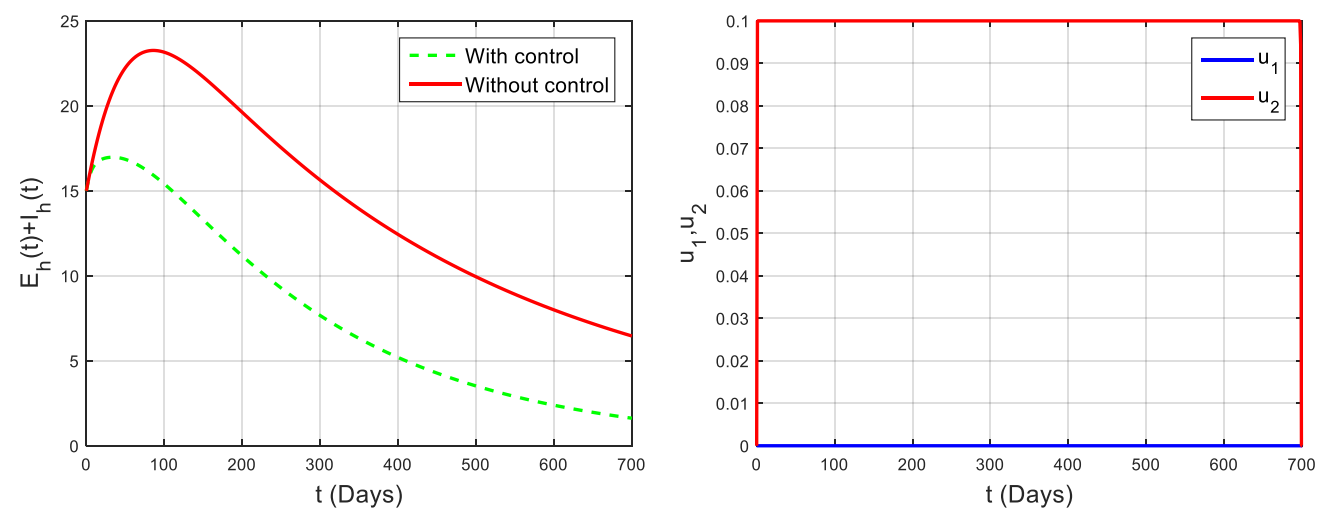

Figure 4. Dynamics of infected human and control profile when strategy 2 is applied

3. Strategy 3: The Effect of Bed-Net Use and Insecticide Intervention.

The effect of the combination of bed-net and insecticide intervention is studied in this subsection. We set both $u_{1} \geq 0$ and $u_{2} \geq 0$. Figure 5 shows that strategy 3 can reduce the number of infected humans. When strategy 3 is applied, the number of infected humans at the end of the period decreases near 0. Figure 5 shows that control $u_{1}$ should be at its upper bound throught 700 days. On the other hand, control $u_{2}$ should be at its upper bound at day $50^{\text {th }}$.
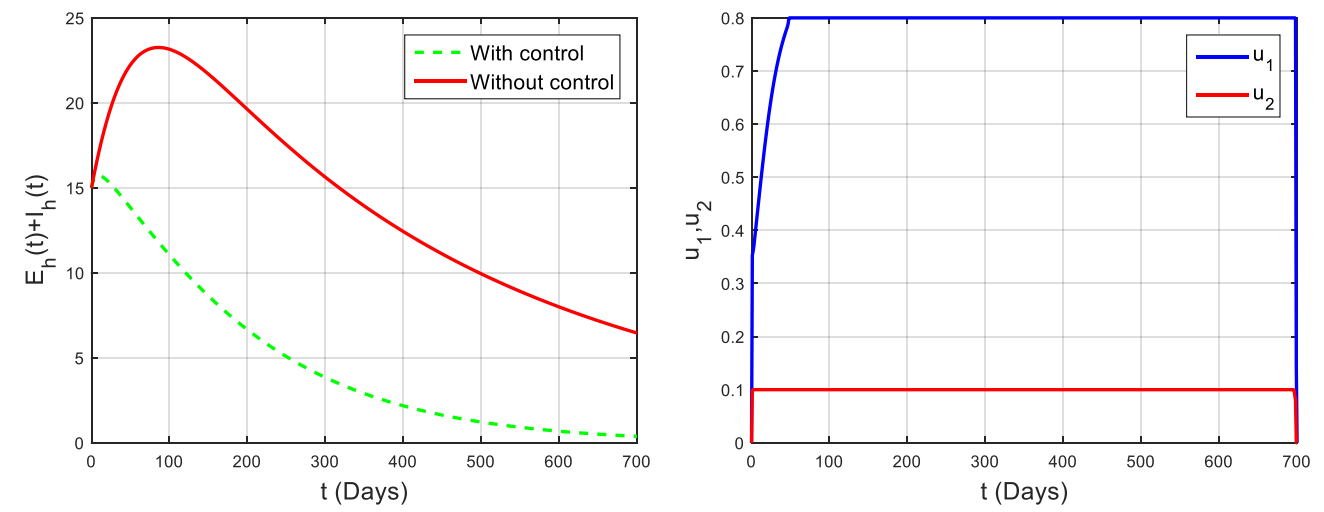

Figure 5. Dynamics of infected human and control profile when strategy 3 is applied

We will find it difficult to determine the most effective strategy in controlling filariasis cases if we only compare the figures and the number of infected humans at the end of the period of each strategy. Here we determine the effectiveness of each strategy using (9). The results obtained are listed in Table 2.

Table 2. Effectiveness of each strategy

\begin{tabular}{cc}
\hline Strategy & Effectiveness $\overline{\bar{E}}$ \\
\hline 1 & 0.67159 \\
2 & 0.46206 \\
3 & 0.66878 \\
\hline
\end{tabular}


It is clear that the most effective strategy is strategy 1 . Now we investigate the most cost-effective strategy using ACER and IAR. We use formula (10) to determine the ACER and IAR of the strategies. The results are given in Table 3. From the ACER

Table 3. Cost-effectiveness of each strategy

\begin{tabular}{cccccc}
\hline Strategy & $T_{a v}$ & $T C$ & Recovered Human & ACER & IAR \\
\hline 1 & 6829.6166 & 446.7419 & 116.0824 & 0.065412 & 58.8342 \\
2 & 4698.7897 & 20.9367 & 123.9002 & 0.0044558 & 37.924 \\
3 & 6800.998 & 455.9003 & 116.2083 & 0.067034 & 58.5242 \\
\hline
\end{tabular}

values presented in Table 3, strategy 2 is the most cost-effective. It is followed by strategy 1 . The least cost-effective strategy is strategy 3 . Therefore, the average cost for each infected human prevented when strategy 2 is employed is lower than when strategy 1 or strategy 3 is implemented. Based on the IAR values listed in Table 3, the most cost-effective strategy is strategy 1 , followed by strategy 3 , then strategy 2 . Hence, the positive impact of curing one infected human on the number of infected humans prevented when strategy 1 is practiced is higher than when strategy 2 or strategy 3 is implemented.

\section{Conclusion}

In this work, we discuss sensitivity, optimal control, and cost-effectiveness analysis of intervention strategies of filariasis. We solve the optimal control problem using Pontryagin's maximum principle. Our findings suggest that the most effective strategy is bed-net use intervention. Using ACER approach, we find that the most cost-effective strategy is insecticide intervention. On the other hand, based on the IAR approach, the most cost-effective strategy is bed-net use intervention. Also, the sensitivity analysis results supports that bed-net use and treatment has a central role in reducing the reproduction number and filariasis spread.

\section{Aknowledgements}

This research is funded by DIPA Universitas Sulawesi Barat, Indonesia, via Penelitian Dasar, in accordance with the Research Contract No. 037/UN55.C/PG/2021, dated 24 May 2021.

\section{References}

[1] B. K. Tyagi, Lymphatic Filariasis. Singapore: Springer, 2018.

[2] T. B. Nutman, "Insights into the Pathogenesis of Disease in Human Lymphatic Filariasis," Lymphatic Research and Biology, vol. 11, no. 3, pp. 144-148, 2013, doi: https://doi.org/10.1089/lrb.2013.0021.

[3] C. T. Goel and A. Goel, Lymphatic Filariasis. Singapore: Springer, 2016.

[4] A. A. Arsin, Epidemiologi Filariasis di Indonesia, 1st ed. Makassar: Masagena Press Makassar, 2016.

[5] A. K. Supriatna, H. Serviana, and E. Soewono, "A Mathematical Model to Investigate the Long-Term Effects of the Lymphatic Filariasis Medical Treatment in Jati Sampurna, West Java," ITB Journal of Sciences, vol. 41, no. 1, pp. 1-14, 2009, doi: https: //doi.org/10.5614/itbj.sci.2009.41.1.1.

[6] C. P. Bhunu and S. Mushayabasa, "Transmission Dynamics of Lymphatic Filariasis: A Mathematical Approach," ISRN Biomathematics, vol. 2012, pp. 1-9, 2012, doi: https://doi.org/10.5402/2012/930130. 
[7] P. M. Mwamtobe, S. M. Simelane, S. Abelman, and J. M. Tchuenche, "Mathematical analysis of a lymphatic filariasis model with quarantine and treatment," BMC Public Health, vol. 17, no. 1, p. 265, 2017, doi: https://doi.org/10.1186/s12889-017-4160-8.

[8] S. M. Simelane, P. M. Mwamtobe, S. Abelman, and J. M. Tchuenche, "A Mathematical Model for the Transmission Dynamics of Lymphatic Filariasis with Intervention Strategies," Acta Biotheoretica, vol. 68, no. 3, pp. 297-320, 2020, doi: https://doi.org/10.1007/ s10441-019-09370-y.

[9] A. J. Iddi, "Lymphatic Filariasis Model with Prevention and Treatment in Human Under Treatment Barriers," International Journal of Scientific Research in Science, Engineering and Technology, pp. 280-292, 2020, doi: https://doi.org/10.32628/IJSRSET207263.

[10] N. Chitnis, J. M. Hyman, and J. M. Cushing, "Determining Important Parameters in the Spread of Malaria Through the Sensitivity Analysis of a Mathematical Model," Bulletin of Mathematical Biology, vol. 70, no. 5, pp. 1272-1296, 2008, doi: https: //doi.org/10.1007/s11538-008-9299-0.

[11] K. O. Okosun, O. Rachid, and N. Marcus, "Optimal control strategies and cost-effectiveness analysis of a malaria model," Biosystems, vol. 111, no. 2, pp. 83-101, 2013, doi: https://doi.org/10.1016/j.biosystems.2012.09.008.

[12] F. Agusto, "Optimal isolation control strategies and cost-effectiveness analysis of a two-strain avian influenza model," Biosystems, vol. 113, no. 3, pp. 155-164, 2013, doi: https://doi.org/10.1016/j.biosystems.2013.06.004.

[13] P. Rodrigues, C. J. Silva, and D. F. M. Torres, "Cost-Effectiveness Analysis of Optimal Control Measures for Tuberculosis," Bulletin of Mathematical Biology, vol. 76, no. 10, pp. 2627-2645, 2014, doi: https://doi.org/10.1007/s11538-014-0028-6.

[14] A. Hugo, O. D. Makinde, S. Kumar, and F. F. Chibwana, "Optimal control and cost effectiveness analysis for Newcastle disease eco-epidemiological model in Tanzania," Journal of Biological Dynamics, vol. 11, no. 1, pp. 190-209, 2017, doi: https://doi.org/10.1080/ 17513758.2016.1258093.

[15] B. Buonomo and R. Della Marca, "Optimal bed net use for a dengue disease model with mosquito seasonal pattern," Mathematical Methods in the Applied Sciences, 2017, doi: https://doi.org/10.1002/mma.4629.

[16] P. Van Den Driessche and J. Watmough, "Reproduction numbers and sub-threshold endemic equilibria for compartmental models of disease transmission," Mathematical Biosciences, vol. 180, no. 1-2, pp. 29-48, 2002, doi: https://doi.org/10.1016/S0025-5564(02)00108-6.

[17] W. Nur, Trisilowati, A. Suryanto, and W. M. Kusumawinahyu, "Mathematical model of schistosomiasis with health education and molluscicide intervention," Journal of Physics: Conference Series, vol. 1821, no. 1, p. 012033, 2021, doi: https://doi.org/10.1088/1742-6596/ $1821 / 1 / 012033$.

[18] E. P. Astuti, M. Widawati, Y. Yuliasih, A. Ruliansyah, and A. J. Kusnandar, "Lama hidup dan potensi culex quinquefasciatus sebagai vektor filariasis limfatik berdasarkan ketinggian pasca transmission assesment survey (tas) di kabupaten subang, Jawa Barat," Vektora: Jurnal Vektor dan Reservoir Penyakit, vol. 12, no. 2, pp. 155-166, 2020, doi: https://doi.org/10.22435/vk.v12i2.3241.

[19] M. Maksud, S. Chadijah, H. Mustafa, A. Kurniawan, and M. Murni, "Keanekaragaman Spesies Nyamuk di Daerah Transmission Assesment Survey (TAS) Filariasis di Kabupaten Enrekang, Provinsi Sulawesi Selatan," BALABA: Jurnal Litbang Pengendalian Penyakit Bersumber Binatang Banjarnegara, pp. 79-90, 2020, doi: https://doi.org/10.22435/blb.v16i1.2814.

[20] W. A. Stolk, S. J. de Vlas, and J. D. F. Habbema, "Advances and challenges in predicting the impact of lymphatic filariasis elimination programmes by mathematical modelling," Filaria Journal, vol. 5, no. 1, p. 5, 2006, doi: https://doi.org/10.1186/1475-2883-5-5.

[21] M. Ismail, R. Jayakody, G. Weil, D. Fernando, M. De Silva, G. De Silva, and W. Balasooriya, "Long-term efficacy of single-dose combinations of albendazole, ivermectin and diethylcarbamazine for the treatment of bancroftian filariasis," Transactions of the Royal Society of Tropical Medicine and Hygiene, vol. 95, no. 3, pp. 332-335, 2001, doi: https://doi.org/10.1016/S0035-9203(01)90257-3. 
Sensitivity, Optimal Control, and Cost-Effectiveness Analysis of Intervention Strategies...

[22] H. A. Farid, S. A. Kamal, G. J. Weil, F. K. Adham, and R. M. R. Ramzy, "Filariasis elimination in Egypt: impact of low microfilaraemics as sources of infection for mosquitoes." Eastern Mediterranean health journal, vol. 9, no. 4, pp. 863-72, 2003.

This article is an open-access article distributed under the terms and conditions of the Creative Commons Attribution-NonCommercial 4.0 International License. Editorial of JoM: Department of Mathematics, Universitas Negeri Gorontalo, Jln. Prof. Dr. Ing. B.J. Habibie, Moutong, Tilongkabila, Kabupaten Bone Bolango, Provinsi Gorontalo 96119, Indonesia. 